\title{
SQUARREL: Scattering Quotient Analysis to Retrieve the Ratio of Elements in X-ray Ptychography
}

Chen-Ting Liao ${ }^{1 *}$, Yuan Hung Lo ${ }^{2,3}$, Jihan Zhou ${ }^{3}$, Arjun Rana ${ }^{3}$, Charles S. Bevis ${ }^{1}$, Guan Gui ${ }^{1}$, Bjoern Enders $^{4}$, Kevin Cannon ${ }^{5}$, David Shapiro ${ }^{4}$, Chris Bennett ${ }^{5}$, Henry Kapteyn ${ }^{1}$, Roger Falcone ${ }^{4}$, Jianwei $\mathrm{Miao}^{3}$, and Margaret Murnane ${ }^{1}$

${ }^{1}$ JILA and Department of Physics, University of Colorado and National Institute of Standards and Technology (NIST), Boulder, CO 80309, USA.

${ }^{2}$ Department of Bioengineering, University of California Los Angeles, CA 90095, USA.

${ }^{3}$ Department of Physics and Astronomy and California NanoSystems Institute, University of California Los Angeles, CA 90095, USA.

${ }^{4}$ Advanced Light Source, Lawrence Berkeley National Laboratory, Berkeley, CA 94720, USA.

${ }^{5}$ Department of Physics, University of Central Florida, Orlando, FL 32816, USA.

*Corresponding author: ChenTing.Liao@Colorado.edu

Coherent X-ray and extreme ultraviolet (EUV) beams can visualize and characterize the structure and functions of material, from tens of microns down to nanometer scales [1,2]. Recent exciting advances from facility-scale synchrotron-based X-ray ptychography has pushed soft X-ray imaging toward $5 \mathrm{~nm}$ spatial resolution [3]; in complementary work, tabletop-scale laser-driven high-harmonic based ptychography has achieved sub-wavelength spatial resolution, achieving $12.6 \mathrm{~nm}$ spatial resolution at a wavelength of $13.5 \mathrm{~nm}$ [4]. Combined with spectroscopic techniques that provide high elemental and chemical specificity, X-ray and EUV spectral imaging can reveal material and biological structures with unprecedented detail to correlate structure-property relations.

Unlike transmission electron microscopy (TEM) based energy-dispersive spectroscopy (EDS), X-ray ptychography alone does not provide the quantitative elemental information needed to extract the composition of a complex sample. This is especially challenging when imaging inhomogeneous materials - where both thickness and composition variations contribute to the change in light absorption and phase retardation. This is because optical density and optical path difference are both the product of the thickness and the complex refractive index of a sample. Here we introduce a new semi-quantitative analysis method in ptychography, named Scattering QUotient Analysis to REtrieve the Ratio of ELements (SQUARREL), to extract elemental information from complex ptychographic images of an inhomogeneous Allende meteorite sample. The resulting maps are especially suitable for studying inhomogeneous specimens, in particular when conventional X-ray and EUV spectroscopic or ptychographic analysis methods fail to extract quantitative information.

In SQUARREL, complex (amplitude and phase) ptychographic images are used to calculate the scattering quotient maps $\left(f_{q}\right)$, which can be defined as $f_{q} \equiv \ln |T| / \phi=\sum_{i} N_{i} \beta_{i} / \sum_{i} N_{i} \delta_{i}$ [5]. $\mathrm{T}$ and $\phi$ are the transmission magnitude and phase retardation images; $\beta$ and $\delta$ are imaginary and real part of the complex refractive index decrement; $N_{i}$ is the number density of the $i^{\text {th }}$ element in the sample. In principle, $f_{q}$ is independent of sample thickness variation, since the thickness has been canceled out in the quotient $[5,6]$. The key to SQUARREL is to convert an $f_{q}$ map into a two-element ratio map, given a fixed amount of a third element as prior knowledge. The conversion function is derived by a direct comparison to a theoretical calculation as a lookup table, which is based on the tabulated complex refractive index database at CXRO [7] of a calculated compound (or mixture in atomic percentage). 
To demonstrate the SQUARREL method, we used the COSMIC soft X-ray imaging beamline at the Advanced Light Source to image a small section of the Allende meteorite, deposited on a TEM grid. The sample is composed of nickel-iron sulfide mixture, which is likely a pentlandite (Fe,Ni) ${ }_{9} \mathrm{~S}_{8}$. The empirical compound formula of the sulfide is $\mathrm{Fe}_{\mathrm{p}} \mathrm{Ni}_{\mathrm{q}} \mathrm{S}_{0.89}$, where $\mathrm{p}+\mathrm{q}=1$. The sample is first pre-characterized using EDS. The experimentally acquired X-ray diffraction images at various photon energies were then scanned and reconstructed by ptychography, to extract the complex images.

Figures 1(a) and (b) show scattering quotient maps from measured complex images using Mg pre-edge $(1295 \mathrm{eV})$ and $\mathrm{Al}$ pre-edge $(1545.5 \mathrm{eV})$, respectively. These maps present a novel image contrast compared to conventional X-ray absorption or phase images. Utilizing the conversion function from SQUARREL, we obtain the converted Fe-Ni ratio maps shown in Fig.2(c) and 2(d), where 100\% implies a pure nickel sulfide mixture without iron. Generally, the converted ratio maps from both pre-edges agree well, despite some small differences, which could be attributed to some uncertainties. The uncertainties include the X-ray photon energy uncertainties, the mixture with a slightly different amount of sulfur, and the inclusion of other elements such as $\mathrm{Al}, \mathrm{Mg}, \mathrm{Si}, \mathrm{C}, \mathrm{O}$. Combining all possible sources of uncertainties, we estimate our total uncertainty is $\sim 20 \%$. In addition, the SQUARREL method relies on tabulated data from the CXRO database, which may have additional uncertainties that were not taken into account [7].

In summary, we introduce a new semi-quantitative method in ptychography, utilizing scattering quotient maps to overcome the elemental quantification problem from an inhomogeneous, varying-thickness sample. Based on the Ni-Fe ratio maps extracted from SQUARREL, the Ni:Fe in our sample is around $1: 1$, which is consistent with the nominal Ni-Fe ratio in pentlandite, validating our assumption of pentlandite mineral phase. An extension of SQUARREL to include more elements without $a$ prior knowledge of the existing third element is also possible and worth exploring in the future. This method could eventually serve as a multi-element ratio quantification and analysis method in X-ray and EUV ptychography, as a complementary analysis tool to EDS, for material and biological sample characterization.

References:

[1] Miao, J., Ishikawa, T., Robinson, I. K. and Murnane, M. M. Science 348, 530-535 (2015).

[2] Pfeiffer, F. Nat. Photonics 12, 9-17 (2018).

[3] Celestre, R. et al. J. Phys. Conf. Ser. 849, 0-4 (2017).

[4] Gardner, D., et al. Nat. Photonics 11.4, 259 (2017).

[5] Jones, M. et al. Sci. Rep. 4, (2014).

[6] Yan, H. et al. Sci. Rep. 6, 20112 (2016).

[7] Henke, B., Gullikson, E., and J. Davis. Atomic Data and Nuclear Data Tables. 54, 181 (1993).

[8] This work was supported by STROBE: A National Science Foundation Science \& Technology

Center, under Grant No. DMR 1548924.
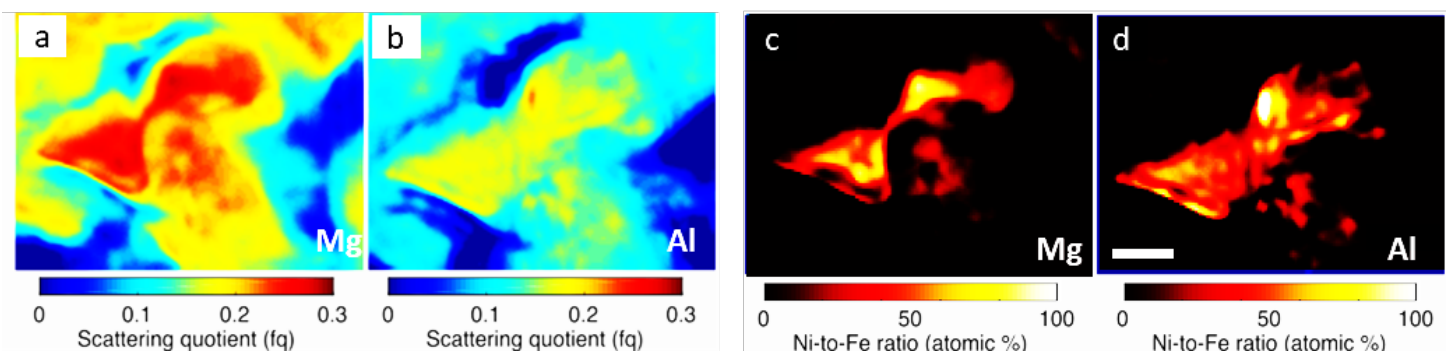

Figure 1. (a) and (b) are scattering quotient maps of a milled Allende meteorite particle, derived from Xray ptychographic $\mathrm{Mg}$ pre-edge and $\mathrm{Al}$ pre-edge complex images, respectively. (c) and (d) are Ni-Fe ratio maps, given a fixed amount of sulfur, converted using the SQUARREL method, where the color bar indicates the Ni-Fe ratio and 100\% implies a pure nickel sulfide region. The scale bar is $200 \mathrm{~nm}$. 